\author{
Pierre Demarque \\ Yale University Observatory
}

\title{
INTRODUCTION
}

It used to be that interest in the ages of the globular clusters was primarily motivated by cosmological considerations. In recent years, remarkable progress in the quality and quantity of observations and advances in the understanding of stellar atmospheres and in the construction of theoretical isochrones make possible the use of globular clusters as probes of galactic evolution. A new picture, still tentative at this point, is emerging of the formation and chemical enrichment of the halo, in its nucleus and in its outer reaches through observations of globular clusters, dwarf spheroidal galaxies and the Magellanic Clouds.

At the same time, the ages of the old open clusters provide crucial information about the formation and age of the galactic disk. old star clusters thus provide necessary input for models of the formation and evolution of galaxies (Ostriker and Thuan 1975; Hartwick 1976; Larson 1976, 1978; Searle 1977; Tinsley and Larson 1978).

\section{THE AGES OF THE NEARBY GLOBULAR CLUSTERS}

The principle of the determination of the ages of the globular clusters from the position of the main sequence turnoff is well known (e.g. Sandage 1970). Normally, the luminosity of the turnoff (or bluest point) from the main sequence on the cluster $c-m$ diagram is compared to the turnoff luminosity on theoretical isochrones with the same chemical composition as the cluster.

From observation one requires: (1) a c-m diagram of the star cluster including the main-sequence turnoff and extending as far as possible down the main-sequence. Corrections for interstellar reddening must be estimated and applied to the cluster. 
(2) the chemical composition of the cluster. Metallicity has so far usually been treated as a one-parameter variable, but this assumption will have to be dropped in the future. The helium abundance $\mathrm{Y}$ is determined independent $1 \mathrm{y}$.

(3) the distance modulus (m-M) of the cluster. There are two possible approaches to determining the distance modulus. The first is to fit the cluster main-sequence to sequence defined by nearby subdwarfs believed to have the same chemical composition as the cluster. The subdwarf distances must be known through trigonometric parallaxes. (Carney (1979b) has made use of this technique most recently.)

The second method is to make use of the known absolute magnitude of RR Lyrae variables. A recent example is that of Saio (1977) who adopted a theoretical formula due to Christy (1966) for the RR Lyrae luminosities as a function of the transition period between fundamental and overtone pulsators. Demarque and McClure (1977) have instead made use of McDonald's (1976) calibration of the luminosities of field RR Lyrae variables.

From (1) and (3), one places the $\mathrm{c}-\mathrm{m}$ diagram in the $\left[\mathrm{M}_{\mathrm{v}}-(\mathrm{B}-\mathrm{V})\right]-$ plane. Since theoretical models are obtained in the [ $\left.\mathrm{M}_{\mathrm{bol}}-\log \mathrm{T}_{\text {eff }}\right]-$ plane, translation from the observational to the theoretical plane must be made through the use of a semi-empirical calibration (Wildey, Burbidge, Sandage and Burbidge 1962) or a grid of model stellar atmospheres (Bell and Gustafsson 1978; Kurucz 1979).

In the past, the fitting procedure has consisted of two steps: (1) the application of blanketing corrections to the observed colors to correct for differences in chemical compositions from normal disk stars (Wildey et al, 1962) and (2) translation of the new colors and magnitudes to the theoretical plane.

A simpler approach, now possible because of recent progress in the construction of model stellar atmospheres, is to work directly in the $c-m$ diagram by converting the theoretical isochrones for a given chemical composition to the $[\mathrm{M}-(B-\mathrm{V})]-p$ lanes (Ciardullo and Demarque 1979), given the surface gravity and effective temperature of each point on the isochrone. This is the technique introduced by Demarque and McClure (1977) and used in the age determinations of Table 1. These ages are based on the Yale isochrones (Ciardullo and Demarque 1977; Sweigart and Gross 1977; Mengel, Sweigart, Demarque and Gross 1979). The clusters M92, M15, M13 and M3 were observed by Sandage (1970), 47 Tuc by Hartwick and Hesser (1977), M5 by Arp (1962), NGC 6752 by Wesselink (1974) and Carnéy (1979a) and M71 by Arp and Hartwick (1971). The reddening corrections are those of Burstein and McDonald (1976). The heavy element abundances are similar to those suggested by Sandage (1970), Canterna (1975), Butler (1975) and Hesser, Hartwick and McClure (1977) and are very uncertain. Figure 1 shows the distribution of ages as a function of $[\mathrm{Fe} / \mathrm{H}]$, together with 
recent age estimates by Hartwick and VandenBerg (1973), Saio (1977) and Carney (1979b) using different fitting techniques which show the uncertainties that remain.

For the small sample of nearby clusters listed in Table 1 , there appears to be a systematic dependence of $[\mathrm{Fe} / \mathrm{H}]$ on age which suggests a relatively slow metal enrichment in this part of the galactic halo. It can be written

$$
\mathrm{t}_{9} \sim-3 .[\mathrm{Fe} / \mathrm{H}]+9,(-2.2<[\mathrm{Fe} / \mathrm{H}]<-0.3)
$$

It must be remembered, however, that uncertainties in the ages due to errors in $[\mathrm{Fe} / \mathrm{H}]$ are significant. A decrease in $[\mathrm{Fe} / \mathrm{H}]$ of 0.4 dex would correspond to an increase in age of about $2.5 \mathrm{Gyr}$. The effects of variations with respect to iron in the relative abundance of helium and elements of the CNO group are discussed in the next section.

TABLE 1

\begin{tabular}{|c|c|c|c|c|}
\hline \multicolumn{5}{|c|}{ AGES OF NEARBY GLOBULAR CLUSTERS } \\
\hline Cluster & $\mathrm{Z}$ & {$[\mathrm{Fe} / \mathrm{H}]$} & $(\mathrm{m}-\mathrm{M})$ & Age (Gyr) \\
\hline M92 & .0001 & -2.1 & 14.49 & $14-16$ \\
\hline M15 & .0001 & -2.0 & 14.90 & $14-16$ \\
\hline M13 & .0004 & -1.4 & 14.50 & $12-14$ \\
\hline M3 & .0004 & -1.5 & 15.03 & $12-14$ \\
\hline NGC 6752 & .0004 & -1.5 & 13.00 & $12-14$ \\
\hline M5 & .001 & -1.0 & 14.40 & $11-13$ \\
\hline 47 Tuc & .006 & -0.5 & 13.38 & $10-11$ \\
\hline M71 & .01 & -0.3 & 13.10 & 10 \\
\hline
\end{tabular}

HELIUM AND CNO ABUNDANCES

Helium abundance. The helium abundance $\mathrm{Y}_{\mathrm{GC}}$ of the globular clusters has attracted special interest because of its importance in cosmology. The value of $\mathrm{Y}_{\mathrm{GC}}$ sets an upper limit on the production of helium in the early universe. And it is also well known that the turnoff luminosity on theoretical isochrones is a sensitive function of $\mathrm{Y}_{\mathrm{GC}}$. In the past only this turnoff luminosity was used as a means of dating the globular clusters without regard to other characteristics of the cluster c-m diagram. Since it is now possible to make a fuller use of the available data and to take into account the fit of the cluster sequence as a whole, it has become clear that only a limited range in $Y_{G C}$ yields an acceptable fit to the observations. In addition to each change in $Y$ corresponds a shift in the position of the main sequence that practically balances out the effect of $Y$ on age. For the oldest clusters, best fit is found for $Y=0.20$, but helium abundances in the range $0.20<\mathrm{Y}<0.30$ seem consistent with the available data. 
PIERRE DEMARQUE

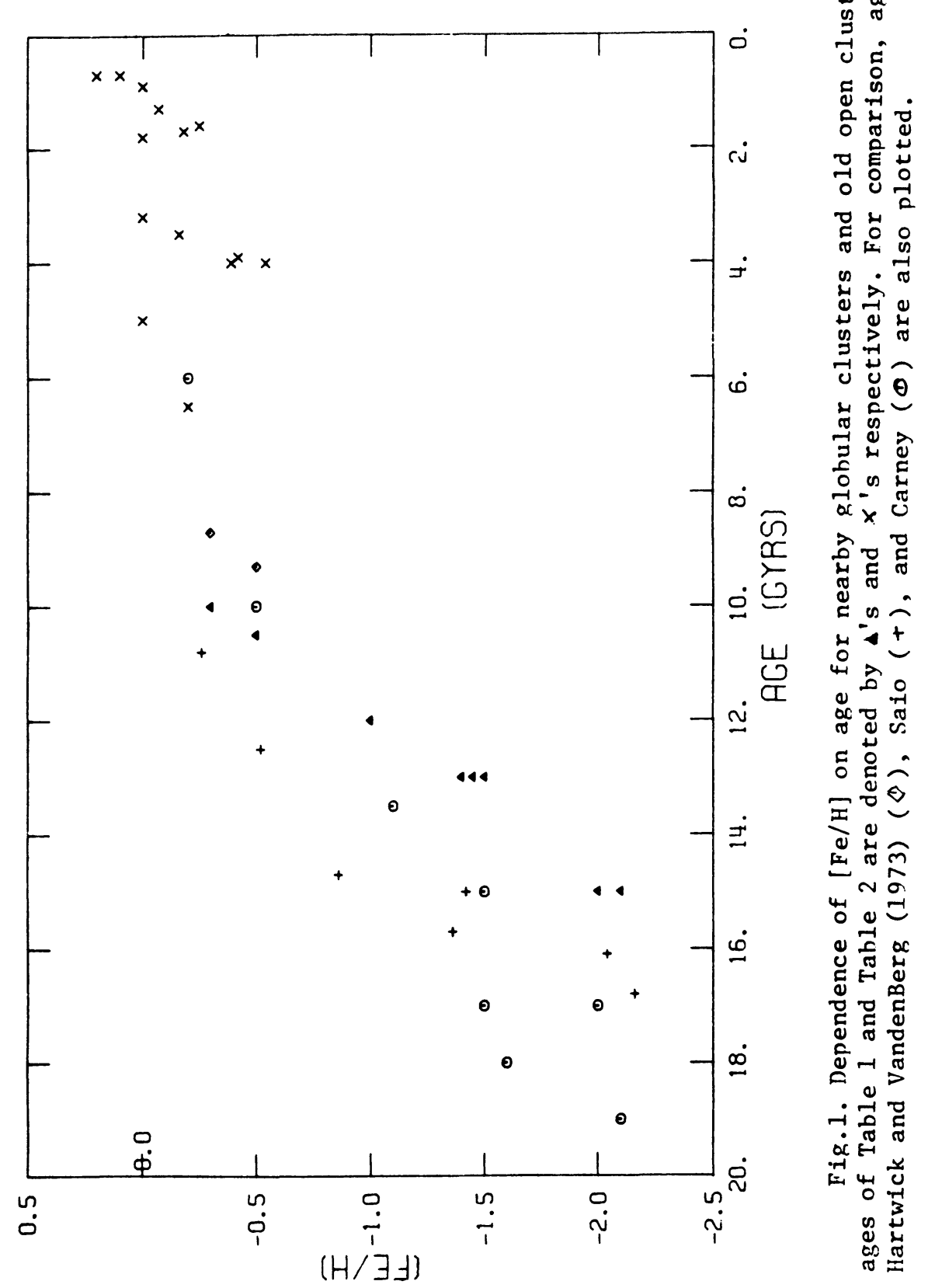


At this point one cannot rule out variations in $\mathrm{Y}$ from cluster to cluster (Green 1979).

CNO abundances. There is evidence for variations in the [CNO/Fe] ratio from cluster to cluster within the galactic halo from observations of CO features in red giants (Cohen, Frogel and Persson 1978; Persson and Cohen 1979). Some field halo stars also exhibit variations in [N/Fe] (Tomkin and Bell 1973) and in particular show an excess in [0/Fe] (Cohen 1978; Tinsley 1979). The importance of the CNO elements in deriving ages for halo stars (Simoda and Iben 1970) and in affecting the colors of horizontal branch stars are well known (Faulkner 1966; Hartwick and McClure 1972; Hartwick and VandenBerg 1973; Castellani and Tornambè 1977).

Tracks of stellar evolution in which the abundances of the CNOelements relative to iron have been varied both in the evaluation of the opacities and in the nuclear energy generation rates have been completed by Rood (1978) and by VandenBerg and Demarque (1979). In the latter calculations the effects of decoupling the $\mathrm{C}$ and $\mathrm{N}$ abundances separately from other heavy elements have been investigated. New opacity tables constructed at the Los Alamos Scientific Laboratory (Cox and Tabor 1977) for $\mathrm{Z}=0.004$ (the same composition as CSVII, CSVIII and CSIX of Cox and Stewart 1970), with the $C$ and $N$ abundances enhanced by a factor of ten respectively. Isochrones for each composition are shown in Figures 2, 3 and 4. The main results for this work are: (1) that the position of the main sequence is unaffected by either change in the $\mathrm{C}$ or $\mathrm{N}$ abundances; (2) that the faint end of the giant branch is also unaffected by the differences in $\mathrm{C}$ or $\mathrm{N}$ abundances; (3) that the main sequence mass luminosity relation is affected, particularly near the turnoff, a given turnoff luminosity corresponding to ages lowered by $10 \%$ and $20 \%$ for enhanced $\mathrm{C}$ or $\mathrm{N}$ respectively. The evolutionary tracks are being extended along the giant branch.

Oxygen is the most abundance of the CNO-elements. There is in addition evidence that the ratio [0/H] increased more rapidly than [Fe/H] during the early phases of galactic evolution (Tinsley 1979). Theoretical work shows the importance of the 0 abundance for horizontal branch morphology through the ON cycle. The effect near the main sequence turnoff of varying the 0 abundance is not as straightforward as for $\mathrm{C}$ and $\mathrm{N}$ since the $\mathrm{ON}$ cycle dominates the energy generation at higher temperatures than the $\mathrm{CN}$ cycle. We note, however, that if $[0 / \mathrm{Fe}]$ is preferentially enhanced for low $[\mathrm{Fe} / \mathrm{H}]$, the ages of the most metal poor clusters would be reduced and the slope of the $[\mathrm{Fe} / \mathrm{H}] \mathrm{vs}$. age relation of Figure 1 would steepen.

\section{OTHER SOURCES OF UNCERTAINTY}

In addition to the uncertainties still existing in opacities, nuclear reaction rates and the physics of convection, there is still a 


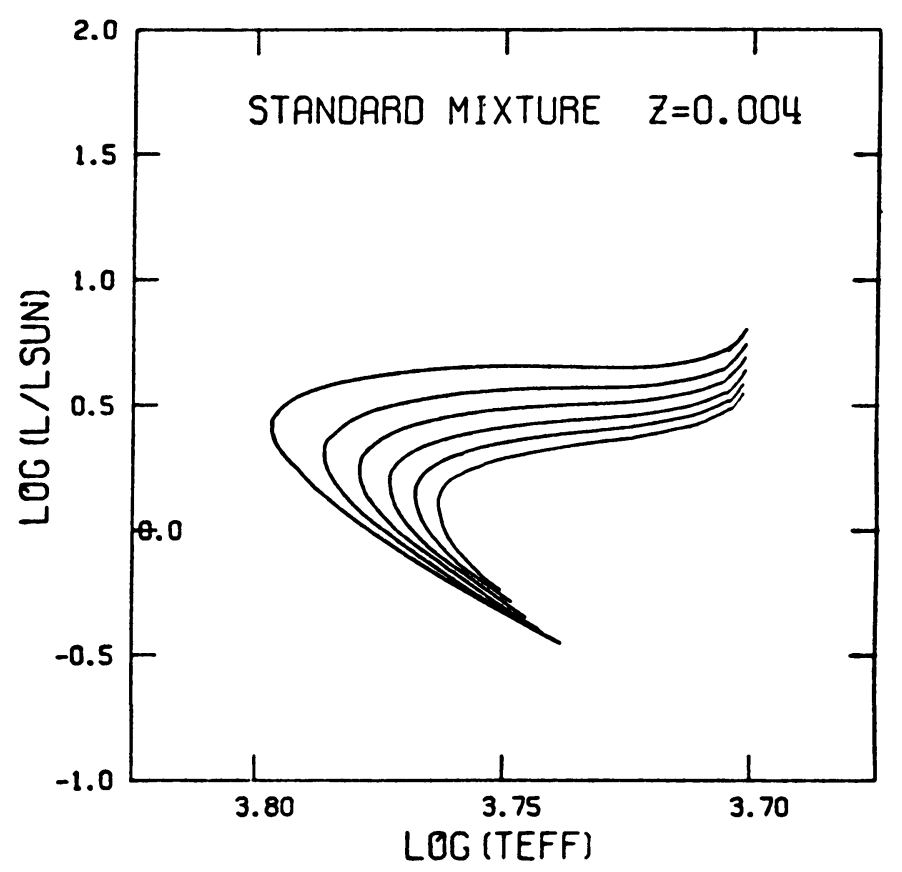

Fig. 2. Theoretical isochrones derived from evolutionary tracks constructed by VandenBerg and Demarque for the compositionparameters $(Y, Z)=(0.25,0.004)$. The interior opacities are due to Cox and Tabor (1977). The relative abundances of the heavy elements with respect to iron are the same as in the standard solar mixture. Each curve represents an isochrone for 8,10,12, 14,16 , or 18 Gyrs respectively. Comparison of Figure 2 with Figures 3 and 4 show that variations in the carbon or nitrogen abundance have 1ittle effect on the morphology of the turnoff. But the ages of clusters are significantly decreased when carbon or nitrogen is enhanced. 


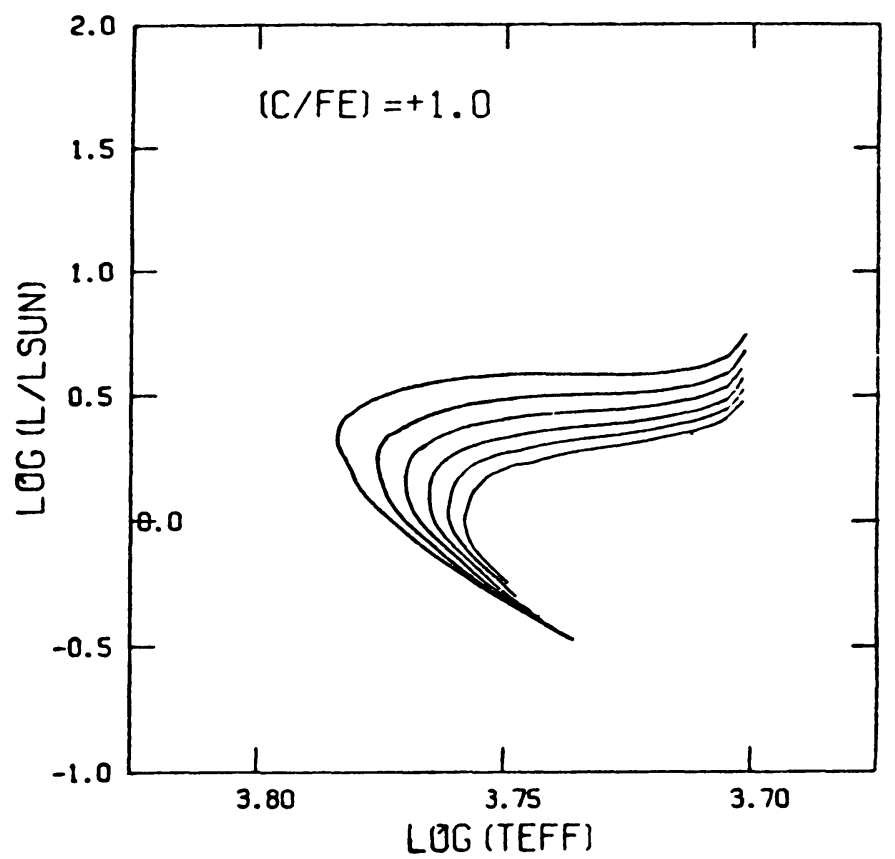

Fig.3. Same as Fig.2, but with enhanced carbon abundance and using the new opacity tables of Cox and Tabor (1977).

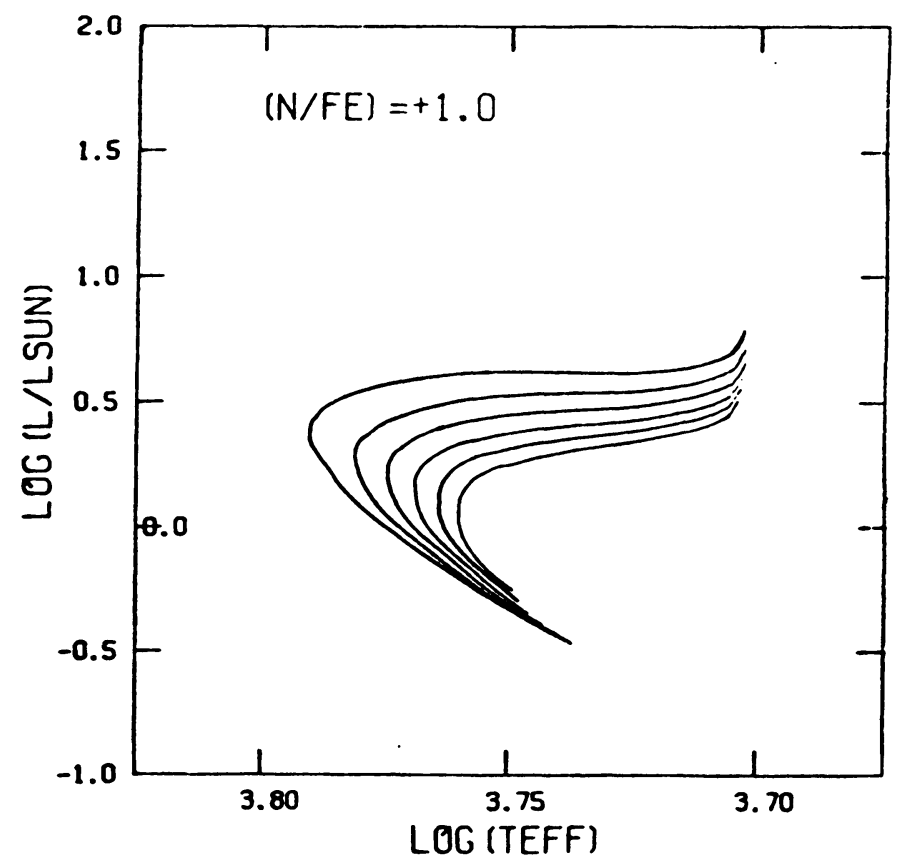

Fig.4. Same as in Figures 2 and 3, with enhanced nitrogen abundance. 
question in some investigators' minds about the reliability of standard models of the kind normally used in age determinations. To list three of the most important one might mention: (1) the possible persistence of internal rotation in the cores of halo stars. Only approximate models for stars with rapidly rotating cores are available (Mengel and Gross 1976) and what the effects of such internal rotation might be on stellar ages is still unclear. Renzini (1977) has discussed in detail the implications of rotating cores on the horizontal branch morphology of globular clusters.

(2) the nature and role of internal mixing. We know that internal mixing beyond that which is expected from standard stellar evolution theory (Iben 1974) takes place in the interiors of at least some red giants. This mixing may be the result of meridional circulation due to rotation (Sweigart and Mengel 1979) or to some still unexplained mechanism.

Similarly the suggestion of the presence of a gap in the c-m diagram of M15 (Sandage and Katem 1977) may be another hint that still unexplained mixing takes place in the interior of stars of low mass.

(3) the possible validity of a non-standard cosmology, such as, for example, Dirac's multiplicative creation theory, an extension of his Large Number Hypothesis (1937), could have a profound effect on the aging processes of stars (Maeder 1977; VandenBerg 1977).

\section{THE AGES OF THE OLD OPEN CLUSTERS}

The old open clusters are important as tracers of the old stellar population of the galactic disk (Patenaude 1978). Their ages, particularly in comparison with the globular clusters, are invaluable in determining a chronology of the formation of the galactic disk. In recent years new discussions of this problem have been made independently by Saio, Shibata and Simoda (1977) and by Demarque and McClure (1977). Both studies agree in concluding that there is a significant age difference between the oldest halo clusters and NGC 188, until recently the oldest known open cluster (McClure and Twarog 1977). There is now and indication that Melotte 66 (Hawarden 1976, Anthony-Twarog, Twarog and McClure 1979) may be a 1ittle older than NGC 188. Table 2 gives a list due to McClure and Twarog (1978) of well observed old open clusters, to which has been added Melotte 66 (the Yale isochrones were used (Ciardullo and Demarque). The clusters are plotted in Figure 1 for comparison with the globular clusters. Two main conclusions emerge from the tabulation: (1) as a group the old disk clusters appear to be several Gyrs younger than M92.

(2) there does not seem to be any correlation between age and metallicity in the available sample. 
These conclusions raise the question of how representative the known open clusters are of the oldest stellar population in the disk, a point discussed by King (1968) several years ago. However, on the basis of the position of the giant branch envelope for disk stars due to Wilson (1976), McClure and Twarog (1978) conclude "that star formation in the disk did not build up to a peak at least until the epoch of formation of NGC 188."

TABLE 2

\begin{tabular}{lrr}
\hline \multicolumn{4}{c}{ AGES AND ABUNDANCES FOR OPEN CLUSTERS } \\
\hline Cluster & Age (Gyr) & {$[\mathrm{Fe} / \mathrm{H}] \mathrm{U}-\mathrm{B}$} \\
\hline & & \\
Hyades & 0.7 & 0.20 \\
NGC 2477 & 0.7 & 0.10 \\
NGC 5822 & 0.9 & 0.00 \\
NGC 2360 & 1.3 & -0.07 \\
NGC 7789 & 1.6 & -0.25 \\
NGC 752 & 1.7 & -0.18 \\
NGC 3680 & 1.8 & 0.00 \\
M67 & 3.2 & 0.00 \\
NGC 6819 & 3.5 & -0.16 \\
NGC 2243 & 3.9 & -0.42 \\
NGC 2506 & 4.0 & -0.54 \\
NGC 2420 & 4.0 & -0.39 \\
NGC 188 & 5.0 & 0.00 \\
Me1 66 & 6.5 & -0.64 \\
\end{tabular}

Finally, one must point out that Table 2 represent a relatively local sample and that the presence of old metal-rich disk stars toward the galactic center cannot be ruled out. In fact one might infer the existence of such stars from the observations of young metal-poor clusters in the anticenter direction (Christian and Janes 1979).

\section{CONSTRAINTS FROM HORIZONTAL BRANCH MORPHOLOGY}

Until we know more about globular clusters, the morphology of the horizontal branch cannot be used for absolute age determination. There are still too many uncertainties in the determination of abundances, in our understanding of mass loss (Fusi-Pecci and Renzini 1975) and other stellar physics. One can use the available information and attempt, however, to place important constraints on globular cluster parameters.

The use of synthetic population models of the horizontal-branch based on stellar models are a powerful tool in research (Rood 1973; Ciardullo and Demarque 1978). The following discussion is based on 

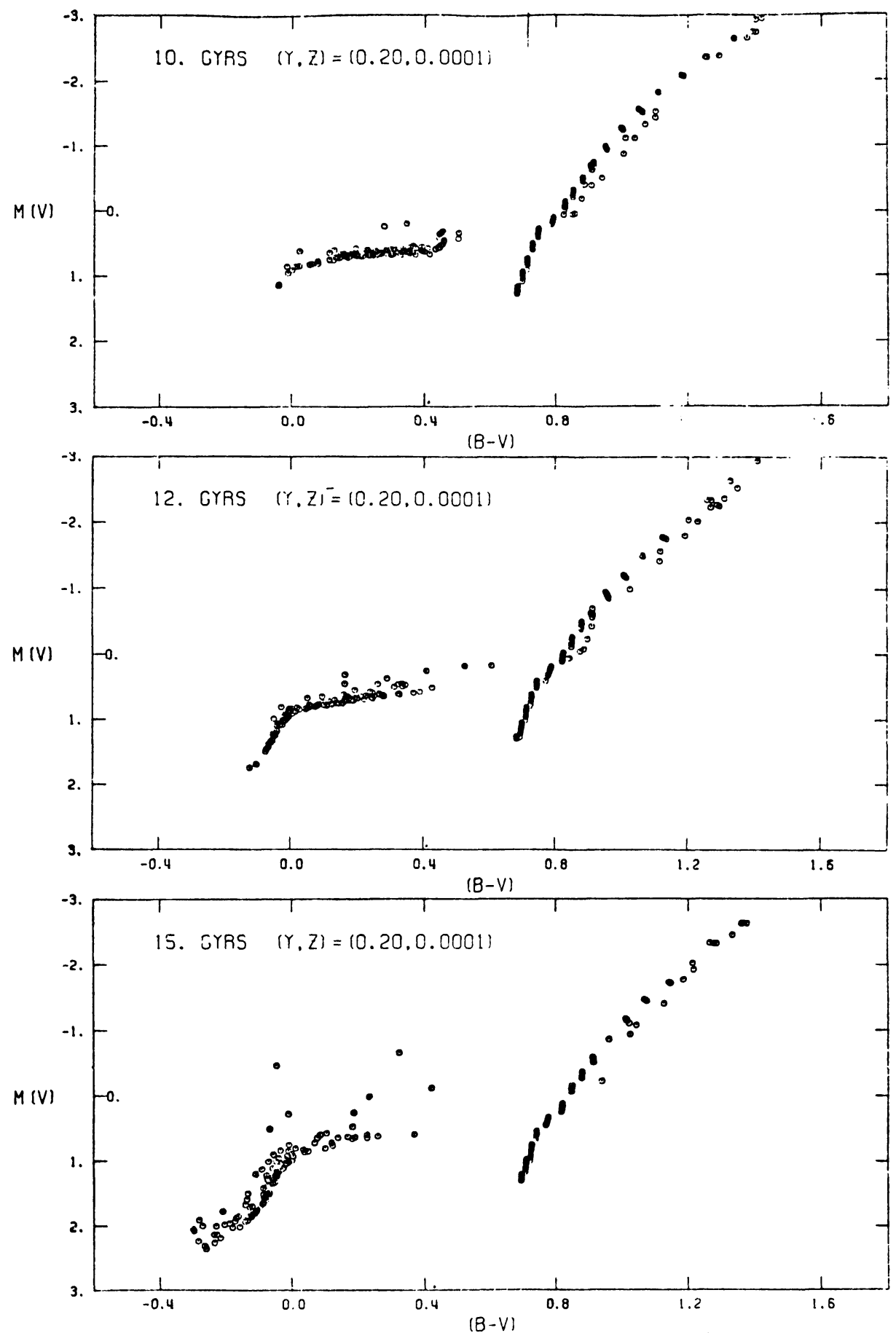

Fig.5. Evolution of the horizontal branch using theoretical stellar models. 

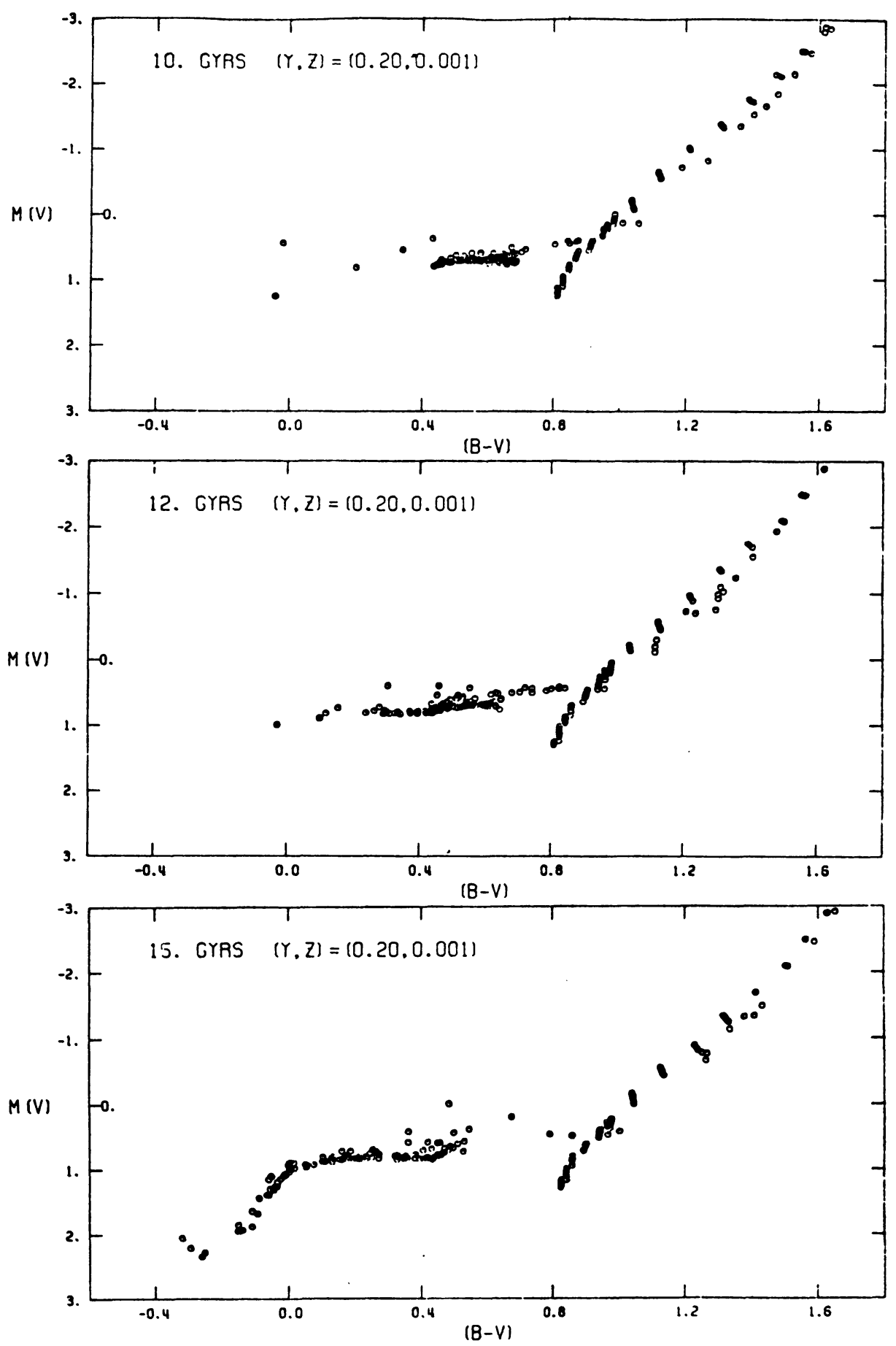

Fig.6. Same as Fig.5 for a higher metal abundance. 


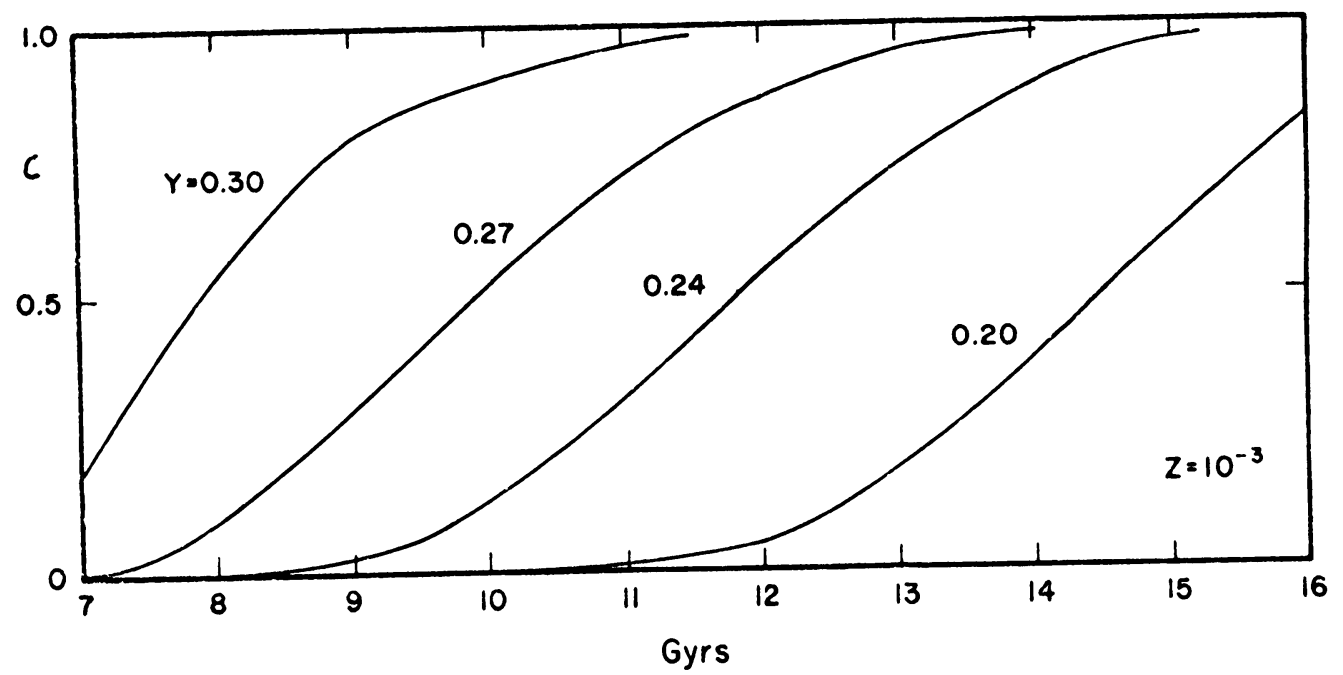

Fig.7. Variation with time of the Mironov index $C$ (Sov. Astron.AJ,16,105,1973) as a function of helium abundance. Due to the uncertainty of the rate of mass loss from red giants, the zero-point on the time axis is poorly defined.

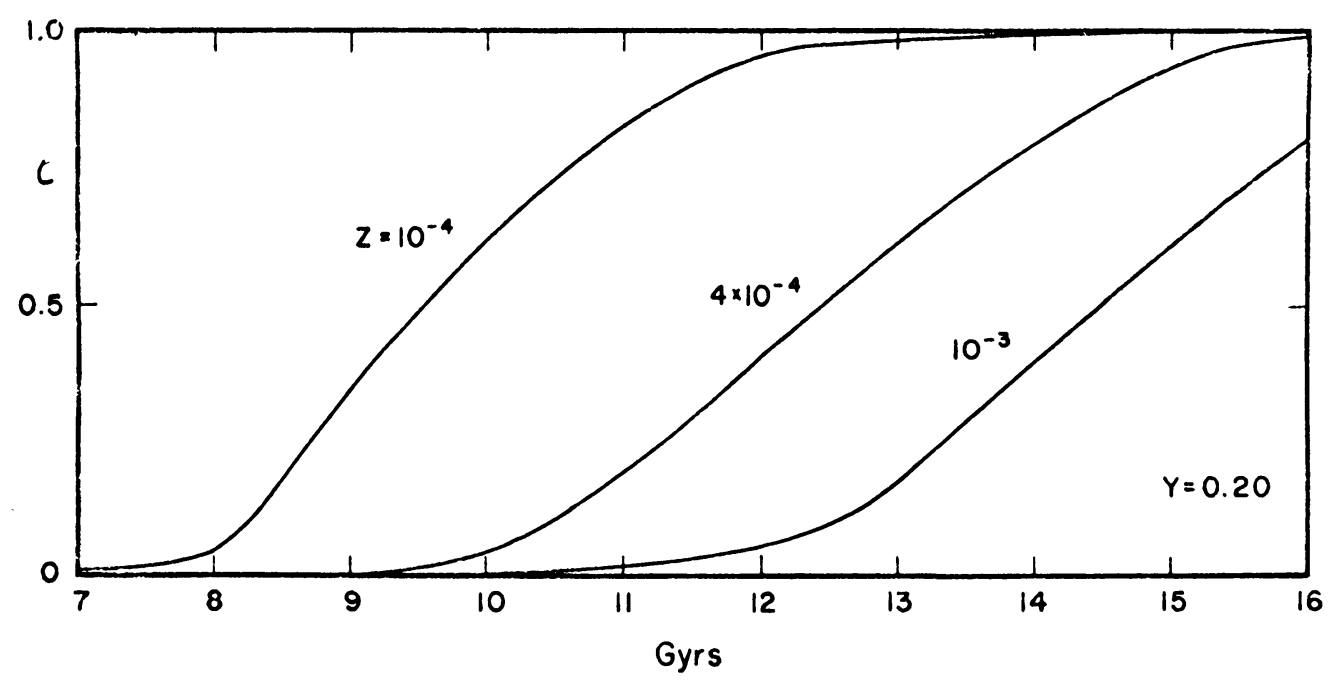

Fig. 8. Variation with time of the Mironov index $\mathrm{C}$ as a function of $\mathrm{Z}$. 


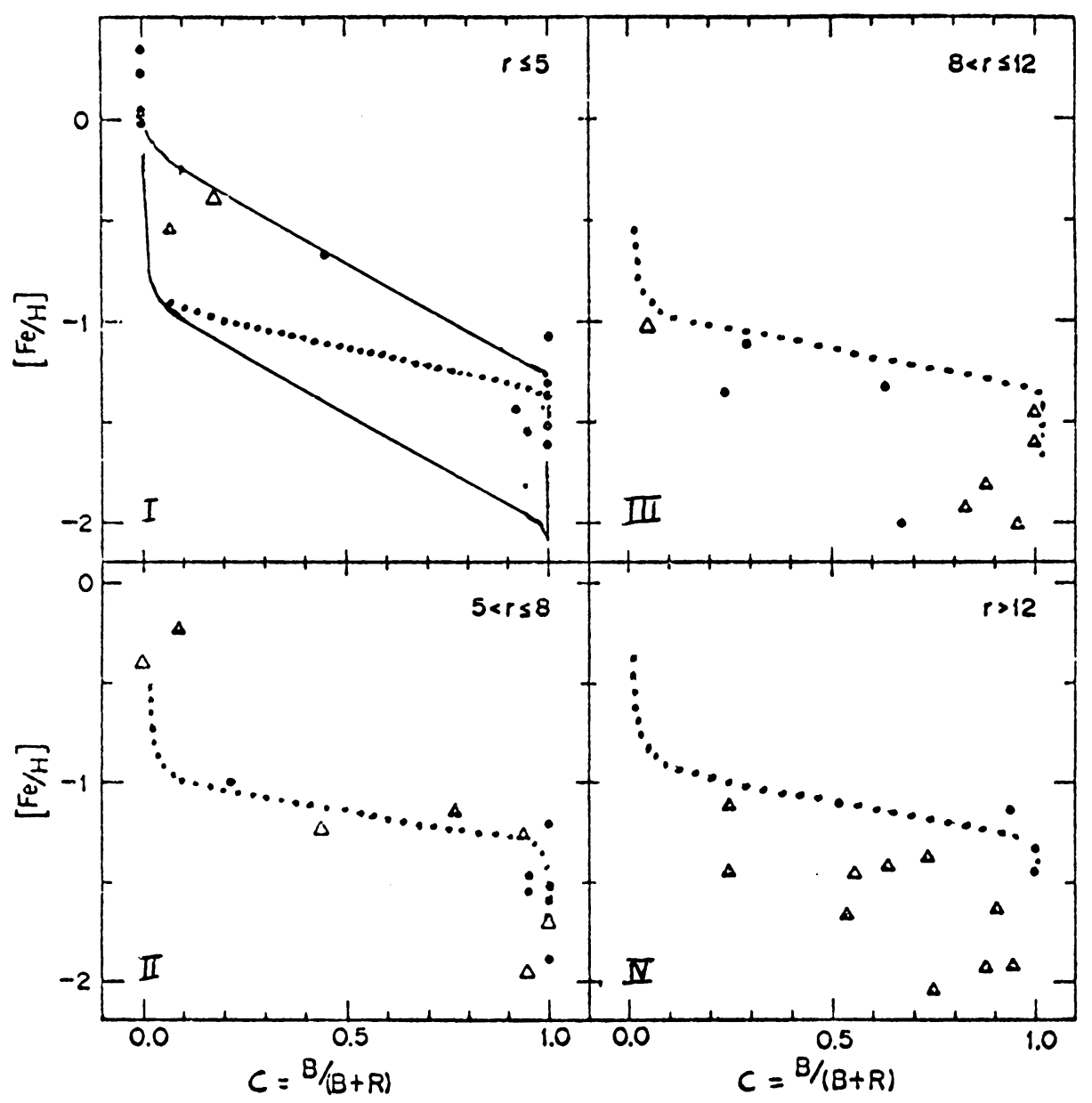

Fig.9. This diagram illustrates the changes in the distribution of globular clusters with different horizontal branch morphology as a function of distance from the galactic center.It is adapted from Fig.10 of Searle and Zinn (1978). 
such models, constructed from tracks of stellar evolution. Some examples are shown in Figures 5 and 6.

Mironov (1973) has defined a convenient index C by

$$
C=\frac{B}{B+R}
$$

where $B$ and $R$ represent the numbers of horizontal branch stars on the blue and red side respectively of the $R R$ Lyrae instability strip in a given star cluster. Figures 7 and 8 show calibrations of $C$ as a function of chemical composition and age based on theoretical calculations. (We note here that the absolute age scale is uncertain since it depends sensitively on the assumed rate of mass loss but that the age differences are reliable).

Now consider Figure 9 which is a plot of [Fe/H] vs $\mathrm{C}$ for globular clusters (Searle and Zinn 1978). In this diagram, a cluster with a given composition describes a horizontal line from 0 to 1 as it evolves. A translation parallel to the abscissa toward the right represents an increase in age. The theory of the horizontal branch tells us that the same displacement could also be caused by (1) a lower CNO-abundance or (2) a higher $Y$. Keeping $Y$ fixed, isochrones can be constructed in Figure 9 using Figure 8 as shown by the solid lines. The difference in age between the two lines corresponds to about 3 Gyrs. If indeed there is a unique relation between the metallicity of globular clusters and their ages in this region, the clusters will fall on a line such as the dotted line in Figure 9. In this line, the metal-poor clusters are older than the metal-rich ones. In the extreme situation of a high sensitivity of age on metallicity, the dotted line is nearly horizontal and most clusters are observed at $\mathrm{C}=0$ or at $\mathrm{C}=1$.

Let us now consider the cluster population in each of the four quadrants of Figure 9 , labelled by Roman numerals. The well-studied "normal" clusters define the dotted line. Clusters that fall off the dotted line are usually called "anomalous". Below the line, we recognize the NGC 7006 anomaly (Sandage and Wildey 1967), above the line the NGC 5053 anomaly (Walker, Pike and McGee 1976).

The dotted line fits well the clusters in quadrant II and forms an upper envelope to the cluster distribution in quadrant III. In quadrant $I$, however, most of the clusters are anomalous, suggesting (barring a systematic overestimate in $[\mathrm{Fe} / \mathrm{H}]$ ) that the most metal-rich ones are older than their counterparts in quadrant II. This result is also consistent with the steep slope of the cluster line in quadrant $I$, pointing to rapid enrichment of the globular clusters within the inner $5 \mathrm{kpc}$ of the Galaxy.

As is well known, the frequency of anomalous clusters (all below the 1ine) in quadrants III and IV (van den Bergh 1967; Searle and Zinn 1978). The upper envelope suggests a slower rate of chemical 
enrichment than toward the galactic center. The data indicate that in the outer halo there is downward scatter in ages of up to 4 Gyrs, or a variable enhancement in total CNO-content, or a spotty decrease in the helium abundance.

\section{THE SECOND PARAMETER}

On the basis of the information discussed so far, there seems to be no clearcut indication for a dominant second parameter. It is not even clear that $[\mathrm{Fe} / \mathrm{H}]$ should remain the "first parameter". We know there are some differences in CNO-abundances. We also know that age differences from cluster to cluster of at least the free-fall time must exist. At this point, we have little knowledge on helium abundances.

The anomalous cepheids do provide an additional piece of information. (Norris and Zinn 1975; Zinn and Searle 1976; Demarque and Hirshfeld 1975; Hirshfeld 1978). The most conservative estimate of the main sequence mass of the anomalous cepheids, based on the mass exchange binary hypothesis (Renzini, Mengel and Sweigart 1977) yields an age of about 9 Gyrs for Draco (Hirshfeld 1978). At least in the case of those systems that contain anomalous cepheids age is the best candidate as "second parameter".

\section{SUMMARY}

On the basis of the fitting of main sequence turnoffs to standard theoretical isochrones, one concludes that:

(1) The ages of the well observed globular clusters (these are relatively nearby clusters with $6<\mathrm{R}<12 \mathrm{kpc}$ ) are in the range $10<t_{9}<15$ Gyrs with an uncertainty of at least \pm 2 Gyrs in each case, mostly due to uncertainties in composition. For these clusters age and metallicity seem correlated, the most metal poor clusters being the oldest.

(2) The ages of the oldest open clusters are in the vicinity of 6 to 7 Gyrs showing a clear gap of several Gyrs between them and the halo clusters. Many of the old open clusters appear to have metal contents as low as the most metal-rich among globular clusters.

The above comments apply to clusters in the vicinity of the sun.

From the horizontal branch morphology compared with stellar models it is possible to draw the following inferences:

(1) All globular clusters within 5 kiloparsecs of the galactic center $(R<5 \mathrm{kpc})$ appear to have closely the same age and to be at 
least as old as M92. This is consistent with a rapid collapse and chemical enrichment among globular clusters near the galactic center.

(2) For $\mathrm{R}>12 \mathrm{kpc}$, globular clusters lie on or below the standard relation for the nearby clusters. There seems to be a downward scatter in ages of up to 4 Gyrs in extreme cases (or excesses in total CNO abundance by a factor of up to five, or deficiencies in $Y$ up to 0.03). Future observations will show which one, if any, of these factors dominate. In the case of systems containing anomalous cepheids, age seems to be an important parameter.

Finally, one cannot emphasize enough the need for reliable abundance determinations of faint stars and for observations of main sequence turnoffs particularly for distant clusters.

This research was supported in part by a grant from the National Science Foundation (AST77-23188).

\section{REFERENCES}

Anthony-Twarog, B. J., Twarog, B. A. and McClure, R. D.: 1979, preprint . Arp, H. C.: 1962, Astrophys. J. 135, 311.

Arp, H. C. and Hartwick, F. D. A.: 1971, Astrophys. J. 167, 499.

Be11, R. A. and Gustafsson, B.: 1978, Astron. Astrophys. Supp1. 34, 229. Burstein, D. and McDonald, L. H.: 1975, Astron. J. 80, 17.

Butler, D.: 1975, Astrophys. J. 200, 68 .

Canterna, R.: 1975, Astrophys. J. (Letters) 200, L63.

Carney, B. W.: 1979a, Astron. J. 84, 515.

Carney, B. W.: 1979b, preprint.

Castellani, V. and Tornambe, A.: 1977, Astron. Astrophys. 61, 427.

Christian, C. A. and Janes, K. A.: 1979, Astron. J. 84, 204.

Christy, R. V.: 1966, Astrophys. J. 144, 108.

Ciardullo, R. B. and Demarque, P.: 1977, Yale Trans., Vol. 33.

Ciardullo, R. B. and Demarque, P.: 1978, in The HR Diagram, ed. A. G. D. Philip and D. S. Hayes.

Ciardullo, R. B. and Demarque, P.: 1979, in Dudley Obs. Report No. 14, ed. A. G. D. Philip.

Cohen, J. G.: 1978, Astrophys. J. 223, 487 .

Cohen, J. G., Froge1, J. A. and Persson, S. E.: 1978, Astrophys. J. $222,165$.

Cox, J. N. and Stewart, J.: 1970, Astrophys. J. Supp1. 19, 243.

Cox, J. N. and Tabor, J. E.: 1977, private communication.

Demarque, P. and Hirshfeld, A. W.: 1975, Astrophys. J. 202, 346.

Demarque, P. and McClure, R. D.: 1977, in The Evolution of Galaxies and Stellar Populations, ed. B. M. Tinsley and R. B. Larson, Yale Univ. Obs.

Dirac, P.: 1937, Nature 139, 323.

Faulkner, J.: 1966, Astrophys. J. 144, 178.

Fusi-Pecci, F. and Renzini, A.: 1975, Mem. Soc. R. Liège, 6th series, 8,383 . 
Green, E.: 1978, This volume, p. 441.

Hartwick, F. D. A.: 1976, Astrophys. J. 209, 418.

Hartwick, F. D. A. and Hesser, J. E.: 1977, Astrophys. J. Suppl. 33,361 .

Hartwick, F. D. A. and McClure, R. D.: 1972, Astrophys. J. (Letters) 176, L57.

Hartwick, F. D. A. and VandenBerg, D. A.: 1973a, Astrophys. J. 185,887 .

Hartwick, F. D. A. and VandenBerg, D. A.: 1973b, Pub. Astron. Soc. Pacific 85,355 .

Hawarden, T. G.: Mon. Not. R. Astron. Soc. 174, 471.

Hesser, J. E., Hartwick, F. D. A. and McClure, R. D.: 1977, Astrophys. J. Supp1. 33, 471.

Hirshfeld, A. W.: 1978, Ph.D. dissertation, Yale University.

Iben, I. Jr.: 1974, Ann. Rev. Astron. Astrophys. 12, 215.

King, I. R.: 1968, Astrophys. J. (Letters) 151, L59.

Kurucz, R.: 1979, Astrophys. J. Supp1. 40, 1.

Larson, R. B.: 1976, Mon. Not. R. Astron. Soc. 176, 31.

Larson, R. B.: 1978, in Chemical and Dynamical Evolution of the Galaxy, ed. E. Basinska-Grzesik and M. Mayor, Torun.

Maeder, A.: 1977, Astron. Astrophys. 56, 359.

McClure, R. D. and Twarog, B. A.: 1977, Astrophys. J. 214, 111.

McClure R. D. and Twarog, B. A.: 1978, in Chemical and Dynamical

Evolution of the Galaxy, ed. E. Basinska-Grezesik and M. Mayor, Torun.

McDonald, L. H.: 1976, Ph.D. dissertation, University of California at Santa Cruz.

Menge1, J. G. and Gross, P. G.: 1976, Astrophys. Space Sci. 41, 407.

Menge1, J. G., Sweigart, A. V., Demarque, P. and Gross, P. G: 1979, Astrophys. J. Supp1. Vo1. 40.

Mironov: 1973, Sov. Astron. J. 16, 105.

Norris, J., and Zinn, R.: 1977, Astrophys. J. 215, 74 .

Ostriker, J. P. and Thuan, T. X.: 1975, Astrophys. J. 202, 353.

Patenaude, M.: 1978, Astron. Astrophys. 66, 225.

Renzini, A.: 1977, in Advanced Stages in Stellar Evolution, Saas-Fe, ed. P. Bouvier and A. Maeder, Geneva Obs.

Rood, R. T.: 1973, Astrophys. J. 184, 815.

Rood, R. T.: 1978, paper presented at the NATO Advanced Study Institute on Globular Clusters, Cambridge, England.

Saio, H.: 1977, Astrophys. Space Sic. 50, 93.

Saio, H., Shibata, Y. and Simoda, M.: 1977, Astrophys. Space Sci. $47,151$.

Sandage, A.: 1970, Astrophys. J. 162, 841.

Sandage, A. and Katem, B.: 1977, Astrophys. J. 215, 62 .

Searle, L.: 1977, in The Evolution of Galaxies and Stellar Populations, ed. B. M. Tinsley and R. B.. Larson, Yale Univ. Obs.

Searle, L. and Zinn, R.: 1978, Astrophys. J. 225, 357.

Simoda, M. and Iben, I. Jr.: 1970, Astrophys. J. Supp1. $22,8$.

Sweigart, A. V. and Gross, P. G.: 1978, Astrophys. J. Supp1. 36, 405.

Sweigart, A. V. and Menge1, J. G.: 1979, Astrophys. J. 229, 624. 
Tinsley, B. M.: 1979, Astrophys. J. $229,1046$.

Tinsley, B. M. and Larson, R. B.: 1978, Astrophys. J. 221, 554.

Tomkin, J. and Be11, R. A.: 1973, Mon. Not. R. Astron. Soc. 163, 117.

VandenBerg, D. A.: 1977, Mon. Not. R. Astron. Soc. 181, 695.

VandenBerg, D. A. and Demarque, P.: 1979, to be published.

van den Bergh, S.: 1967, Astron. J. 72, 70.

Walker, M. F., Pike, C. D. and McGee, J. D.: 1976, Mon. Not. R. Astron. Soc. 175, 525.

Wesselink, A. J.: 1974, Mon. Not. R. Astron. Soc. 168, 345.

Wildey, R. L., Burbidge, E. M., Sandage, A. R. and Burbidge, G. R. 1962, Astrophys. J. 135, 94.

Wilson, 0. C.: 1976, Astrophys. J. 205, 823.

Zinn, R. and Searle, L.: 1976, Astrophys. J. 209, 734. 


\section{DISCUSSION}

ROSSI: I have a question on a point that is not clear to me: do the isochrones depend on the metal abundance? If so, you match your experimental data to the isochrones of a given $Z$ and find the age to be related to $[\mathrm{Fe} / \mathrm{H}]$. Does this procedure already contain implicitly the dependence of metallicity on the age?

$D E M A R Q U E:$ Well, first I get the metallicity from observation. Then knowing the metallicity, one compares the turnoff to the isochrones of that particular metallicity. Then one gets an age and then one can plot age versus metallicity.

ROSSI: I expect that this relation must appear, because when I fit the isochrone I fit a metallicity itself, because the isochrone depends on the metallicity.

VAN DEN BERGH: Taking into account all of the uncertainties that you mentioned, what would you feel are the $2 \sigma$ upper and lower age limits for the oldest globular clusters? (Laughter.)

DEMARQUE: Upper and lower? For the oldest - oh, I don't know; on the basis of this I guess it's on the order of $20 \times 10^{9} \mathrm{y}$. It's just a guess. Maybe even lower -13 x $10^{9} \mathrm{y}$.

RENZINI: I think that the uncertainty in the cluster ages as derived from the turnoff fitting or any way using the turnoff luminosity, is around $4 \times 10^{9} \mathrm{y}$. We cannot do better than this simply because an uncertainty of a quarter of magnitude in the turnoff luminosity means $2.5 \times 10^{9}$ y uncertainty in the age. You couple this with an uncertainty in helium and you end up with about $4 \times 10^{9}$ y uncertainty in the derived ages. Therefore, I will not be surprised with age differences due to the noise of the order of a few billion years, and I am not surprised that, for instance, you find the trend with metallicity. That's because this could well be produced by some systematic effect in the method which is used in deriving the ages when you change the metal abundance. This can happen quite naturally. Suppose you take the RR Lyrae luminosity as constant, $0.6 \mathrm{mag}$, irrespective of metallicity. - Therefore you find a trend, but this trend will be due entirely to the fact that the RR Lyrae luminosities are not exactly the same: suppose they depend slightly on the metallicity. In any other methods you can use you may always have these systematic trends. So, I would say that the cluster ages derived from the turnoff luminosity just tell us that the globular clusters are, say, $(14 \pm 4) \times 10^{9} \mathrm{y}$, and no more than that. It's absolutely impossible with the present data to get more than that. And another point concerning the horizontal branch morphology. The slope of the lines that were shown, say this index, $C$, versus metallicity, also depends on the trend of the mass loss with metallicity. Since we don't know exactly a priori how much mass is lost during the red giant branch evolution as a function of metallicity, you can get any slope changing this. 
DEMARQUE: I agree a $100 \%$. Everything I've shown implies all kinds of assumptions. And, actually, you're right about the ages. The dependence of age on metallicity, I feel, is still quite possible, but the difference in age between the most metal rich and the most metal poor may be just a billion years - we can't tell. However, the dependence of the RR Lyrae luminosity would have to be very large, because Saio's calibration took that into account and, actually, the effect is larger in this case; and I think this is true of Carney's, too.

MAEDER: Your results concerning the trend of the morphology of the horizontal branch with age and chemical composition certainly have very interesting consequences for the evolutionary correction in the Hubble-Sandage diagram for first-ranked galaxies. Would you point out the relevance of your calculation to that problem?

DEMARQUE: Wel1, I'm not exactly sure which particular point you're referring to, but the whole idea, of course, of getting involved in the population models of globular clusters was to eventually be able to find the discriminants to study the integrated colours of such systems. But one has to do it together with studies of the nearby objects in order to understand what one is doing. I don't know exactly what in particular you're referring to in the case of the evolutionary correction.

MAEDER: Usually in calculations of the evolutionary corrections for galaxies you must take into account the change of the main sequence turnoff and you must make some guess about the behavior of the red giants, but here the calculation of the change of the horizontal branch also has very important consequences for the change of the colour and brightness of the first-ranked galaxies. These affect the corrections to the Hubble-Sandage diagram.

$D E M A R Q U E$ : Qualitatively, if you plot the evolution of a very simple galaxy in this colour-colour plane, instead of the usual evolutionary correction, you get at some point during the life of the system a point which is probably relevant for the observation of, at least, some of the galaxies we've observed with ages between 10 and $20 \times 10^{9} \mathrm{y}$. You get a loop or a hook due to the horizontal branch as it passes across the HR diagram. of course, this is very important for evolutionary corrections. If we happen to observe galaxies in this region we cannot apply the usual evolutionary corrections.

BOK: I would like to ask two slightly related questions. The first one is, you seem to place a great deal of confidence in the theoretically computed absolute magnitudes of RR Lyrae stars. Would you tell us briefly how these compare with the calibrations that have been made? The second point is that among the Cepheids we have at the moment this problem that plagues the Magellanic Clouds: the Cepheids give a smaller distance than the RR Lyraes. Is your theoretical approach possible to extend? Will that help us to settle the business how the long-period Cepheids and the RR Lyraes get together? 
DEMARQUE: I did not make myself clear. There is nothing theoretical in the determination of the RR Lyrae magnitudes that I've used. I used a value of $0.6 \mathrm{mag}$, partly because observationally it seems to be a good value and in the Magellanic Clouds it seems to be a good value, and then there is the study by . . .

$B O K$ : Not if you talk to Clube! I've been subjected to Edinburgh.

DEMARQUE: Well, there is also a study by McDonald, using the Wesselink method, which is a good physical method. He suggests, at least for the field RR Lyrac stars having different metallicities within a reasonable range, you get more or less the same absolute magnitude - around 0.6. I tend to prefer those techniques - this is my bias - which involve some sort of physical model.

WALLERSTEIN: While it's unfortunate to anticipate some of the results to be given tomorrow afternoon, it's even more unfortunate that some people will have to be leaving before those results on abundances are given; and because this has a very substantial effect on the diagram of Searle and Zinn that you showed. The most metal-rich, upper-left-hand-corner globulars are calibrated on the basis of 47 Tucanae and M71 and we'11 be hearing tomorrow afternoon that they're not nearly as metal rich as people have said in the past. They have more nearly $[\mathrm{Fe} / \mathrm{H}]$ around -1 or -1.2 , which brings them right down with the rest of the boys. Also, the parameter that should be plotted, as you said but it should be even more emphasized, is not really the iron abundance but the $\mathrm{C}+\mathrm{N}+\mathrm{O}$ abundance, because those are the elements which participate in the CNO cycle and therefore determine the ratio of burning in the shell to the ratio of burning in the core. Substantial evidence by Cohen and Pilachowski, who will be telling us all of this tomorrow, that amongst the clusters of intermediate metal abundance the ones with strong $\mathrm{CO}$ and the ones with strong oxygen, which can be done independently by completely different methods, do in fact correlate as the ones which have the rather red horizontal branches. When the parameters are used that way, then, I think that it changes the morphology of those diagrams very substantially.

COHEN: I'l1 agree with the first part of George's statement, but I think in Jay Frogel's talk tomorrow afternoon there will be a reasonable amount of evidence presented which may not convince everybody but certainly casts some doubt on our previous suggestion that. the CNO elements are actually the second parameter. I urge you to come listen.

WALLERSTEIN: All flights at noon tomorrow will be cancelled!

CARNEY: Just a quick question here. Did you ever calibrate your isochrone models against the Sun to derive a mixing length and helium abundance?

DEMARQUE: Yes; well, not the helium abundance. The helium abundance you get from the Sun is a little higher. It's more like 23 or 24 per cent by mass. 
CARNEY: That's about what Iben and Mahaffy got when they used their code to calibrate against the Sun. They got a mixing length of 1 and $\mathrm{Y}=0.23$.

DEMARQUE: It would be higher than 1 with our models.

CAYREL: To come back to Dr. Renzini's discussion about his mean age of $14 \times 10^{9} \mathrm{y}$. I'm a little afraid that this plus or minus of $4 \times 10^{9}$ y will set the lower limit of the galaxy too low, at $9 \times 10^{9} \mathrm{y}$, which is almost not admissible. But if you gave the oldest disc population the mean age of the subgiants, which is 20 , between 18 and $24 \times 10^{9} \mathrm{y}$, then you can put the $2 \sigma$ error bars of, let us say, $8 \times 10^{9}$ y and you go down only to $15 \times 10^{9} \mathrm{y}$ as the lower limit of the age of the galaxy Philosophically I would prefer this lower limit, and not $9 \times 10^{9}$ y. (Laughter).

DEMARQUE: I understand that some people are interested in the determination of the Hubble constant. (Laughter).

KRAFT: I'd like to ride a little hobby horse that I've ridden in the literature several times and that is to call attention once again to the existence of the metal-rich RR Lyrae stars in the old galactic disc. These stars constitute about $25 \%$ of the total population of RR Lyrae stars in the original Preston survey. My question is, where do they come from? If the old galactic disc is really no more than 5 , or 6 or $7 \times 10^{9}$ y old and those stars come out of that, then they must be mapped from stars whose masses are about $1.1 \mathrm{M}_{\odot}$, or something like that. And that means that in order to get into the instability strip they have to loose about $0.5 \mathrm{M}_{\odot}$, and I don't think any theory, Renzini's or anybody else's, on mass loss in the giant star domain can probably pump that much mass loss in those stars. So, as Renzini has pointed out, it's a very tricky business how you get over a horizontal branch at all, and you've got to loose just the right amount of mass. So, if the mass loss is of the order of 0.1-0.2 $\mathrm{M}_{\odot}$, you're not going to get into the RR Lyrae domain or horizontal branch. The more comfortable solution, it seems to me, is to admit that there is an older aisc component of metal-rich stars whose ages are such that the turnoff mass can be, let's say $0.8 \mathrm{M}_{\odot}$, and that would tend to give one at least some component of the age of the galactic disc that is metal rich and that is considerably older than the clusters that you were talking about.

DEMARQUE: What is the metallicity of these?

KRAFT: The mean metallicity is about -0.3 or -0.4 . It's the same as Eggen's old-disc population giants.

KEENAN: Perhaps I can make a comment about that. Sandage, of course, mentioned that a fair while ago too, about those old metal rich RR Lyrae stars. How would you think about the idea that they possibly came from a disruptive population of old disc globulars? Van Albada and some other people have worked on this. Possibly an old disc population of globulars disrupted and dumped these stars. 
GASCOIGNE: There's a note in Astronomy and Astrophysics by van Albada and Dickens in which they reported on some ultraviolet observations of the integrated light of the galactic globular clusters. Those give us a very accurate age and the age scatter is quite small, possibly only about $10^{9} \mathrm{y}$. It works because basically they are looking at the stars near the turnoff, and I'm just wondering if you have seen this and, if so, what you think of it?

DEMARQUE: Yes, I've seen it and it's a little alarming, actually, because they use the same data. They use our data, our luminosity functions and isochrones, to derive those ages. I haven't seen the details of their calculations, but it shows that using the same theoretical evolutionary tracks, which of course is a similar case for the blue stragglers, you'll get quite a difference, really, in calibration for the ages. Depending on how one uses that, one can get quite a wide variety of answers.

KING: I have a comment and a question. The comment is on the photometry. I was rather shocked to see the spread in the photometric points compared to the very small difference that corresponds to $2 \times 10^{9}$ y in age; and I really wonder, and I wonder out loud in the presence of observers, whether that photometry can't be improved for the near, easy clusters by choosing stars carefully and taking the stars for which you know you can get really accurate photometry? If the spread remains it's astrophysically interesting; if the spread goes away, then maybe you produce numbers that you have to stand behind. (Laughter). The question is, I think, a naive one. You've talked about the colour of the turnoff. You haven't talked about it's shape. Now the shape and the differences in shape of turnoff transition from the main sequence to the subgiants branch are quite striking. Are they completely explained by metal abundance, and also do the numbers help at all or do you fit perfectly well with evolutionary rates?

DEMARQUE: Again, the shapes were actually used in the fitting more than the actual colours. We tend not to fit on the colour, which is, incidentally, my one bone of contention with Carney. The colour is quite uncertain because of the mixing length problem, which makes the radii uncertain. If you look at different evolutionary tracks by different authors you find that usually the agreement between the change in luminosity with time is very good, but we do differ quite a bit in colours and the effective temperatures of the models. When you talked about the numbers, are you referring to a luminosity function, really?

KING: Yes.

DEMARQUE: No, I guess we haven't used a luminosity function. That should be another piece of information, but I think the colourmagnitude diagrams really don't give us any idea of the luminosity function, because they just take a selection of stars. So we have to go back and look at other work on the luminosity function.

MCCLURE: I think we better move on or we're not going to get any lunch. 